\author{
Submitted: \\ 21.10.2020 \\ Accepted: \\ 17.12.2020 \\ Published: \\ 08.03.2021

\section{The diagnostic value of ultrasound-guided percutaneous core needle biopsy of musculoskeletal soft tissue lesions} \\ Paweł Szaro',2, Andrew Wong ${ }^{3,4}$, Elena Blain, \\ Khaldun Ghali Gataa ${ }^{4}$, Mats Geijer ${ }^{1,5}$ \\ ${ }^{I}$ Department of Radiology, Institute of Clinical Sciences, Sahlgrenska Academy, University \\ of Gothenburg, Sweden \\ ${ }^{2}$ Department of Descriptive and Clinical Anatomy, Medical University of Warsaw, Poland \\ ${ }^{3}$ Department of Radiology and Nuclear Medicine, Radboud University Medical Center, \\ Nijmegen, Netherlands \\ ${ }^{4}$ Department of Musculoskeletal Radiology, Sahlgrenska University Hospital, Sweden \\ ${ }^{5}$ Department of Clinical Sciences, Lund University, Sweden \\ Correspondence: Pawet Szaro, Department of Radiology, Institute of Clinical Sciences, \\ Sahlgrenska Academy, University of Gothenburg, Gothenburg, Sweden; \\ e-mail:pawel.szaro@gu.se
}

Keywords

ultrasound,

biopsy,

solid tumor,

musculoskeletal

system,

diagnostic biopsy

DOI: $10.15557 / \mathrm{JoU} .2021 .0004$

\begin{abstract}
Introduction: Percutaneous ultrasound-guided core needle biopsy is a well-established method in the diagnosis of musculoskeletal tumors. It is unclear which factors contribute the most to a successful biopsy. The aim of the study was to determine the value of ultrasoundguided core needle biopsy of solid lesions in the musculoskeletal system using a 16-gauge needle. Material and methods: A retrospective analysis performed at a regional sarcoma center over one year included patients referred for ultrasound-guided biopsy of musculoskeletal soft tissue lesions. At least 6 months' clinical and radiological follow-up, results from repeat or excisional biopsy, or interventional treatment, served as outcome reference. The biopsy procedure and yield were analyzed. The biopsy was classified as 'diagnostic' when a definitive diagnosis could be made on the first biopsy, and 'accurate' when only the malignant or benign nature of the tumor could be determined. Results: From 102 referrals for biopsy of soft tissue lesions in 2019, a total of 73 biopsies of solid lesions with a 16-gauge cutting needle were included (73 patients). There were 34 males and 39 females, with a mean age of 57.7 years. The overall proportion of diagnostic biopsies was $84 \%$, for malignant lesions $88 \%$ and benign lesions $81 \%$. The tumor could be classified as malignant or benign in 12 patients (16\%) (accurate biopsy). It was possible to discriminate between malignant and benign lesions in each case. The majority of biopsied lesions were benign $64 \%(n=47)$. Conclusion: The diagnostic value of ultrasound-guided percutaneous core needle biopsy of musculoskeletal soft tissue lesions performed with 16-gauge needle is good, with a high rate of diagnostic biopsies, both for benign and malignant lesions.
\end{abstract}

\section{Introduction}

The advantages of ultrasound-guided biopsy for the diagnosis of musculoskeletal tissue lesions are wellknown ${ }^{(1-3)}$. A modern approach to diagnosis and the importance of the multidisciplinary tumor board in patient survival have been emphasized ${ }^{(4-6)}$. Diagnostic imaging with radiography, magnetic resonance imaging (MRI), computed tomography (CT), and ultrasonography is helpful in the process of differential diagnosis. It is, however, difficult in most cases to sufficiently narrow the range of differential diagnoses of a soft tissue lesion using radiology alone. A histopathological verification may be necessary in cases where the 
radiological signs are not typical or the clinical presentation is inconsistent with the radiological findings ${ }^{(7)}$. Ultrasound-guided core needle biopsy (US-CNB) is performed not only to make the diagnosis, but also to assess tumor viability, which helps to choose the optimal treatment. Biopsy accuracy has been reported as ranging between $60 \%$ and $97 \%$ for ultrasound-guided biopsy. It varies between reports because of different indications, different selection of needles (fine or core needle) and different selection of lesions ${ }^{(1,3,8-14)}$. The success of a biopsy - measured by the ability of establishing a histopathological diagnosis from a tissue sample - may depend on the selection of patients, so multidisciplinary cooperation is necessary ${ }^{(7,13)}$.

US-CNB is a safe procedure, without major complications. Pain, bleeding, needle breakage, nerve or vessel injury, or pneumothorax, occur rarely $(0.5-10 \%)^{(7,14)}$. The rate of complications after surgical or excisional biopsy has been reported as higher than for US-CNB $(8-17 \%)^{(2,9,12,13)}$. It is possible to improve the safety of the biopsy procedure by using ultrasound guidance with color Doppler, thus avoiding injury to major vessels or nerves adjacent to the tumor $^{(7,8)}$. The risk of neoplastic seeding in the needle tract is much lower compared to surgical biopsy, the rate being reported at approximately $0,003-0,005 \%$ or not observed at all $^{(8,13,15,16)}$.

The current study aimed to assess the value of ultrasoundguided biopsy of solid tumors in the musculoskeletal system using a 16-gauge needle.

\section{Material and methods}

\section{Patients}

The study site is a university hospital with a dedicated multidisciplinary team concerned with the diagnosis and treatment of bone and soft-tissue sarcomas. In a retrospective analysis, a total of 102 referrals were received for ultrasound-guided biopsy of a musculoskeletal soft tissue lesion during 2019. The exclusion criteria were fear of medical procedures involving needles (4 patients), purely cystic lesions (6 patients), and biopsy of lymph nodes (5 patients) (Fig. 1). Further 12 patients were excluded from the study since their biopsy was performed under CT guidance. Ultimately, 73 patients with a primary US-CNB of a soft tissue lesion in the musculoskeletal system were included. The group of 73 patients included 34 males and 39 females, with a mean age of 57.7 years (range 8-89). The most common localization of lesions was the lower extremity, followed by the upper extremity. The patients were followed up for at least 6 months $(n=49)$, had repeated biopsies, underwent interventional procedures with expected good effects $(n=2)$, or had surgical treatment $(n=19)$.

\section{Biopsy procedure}

All the procedures were performed using a LOGIQ E9 (General Electrics) ultrasound scanner with a linear or convex transducer, as appropriate. The shortest distance

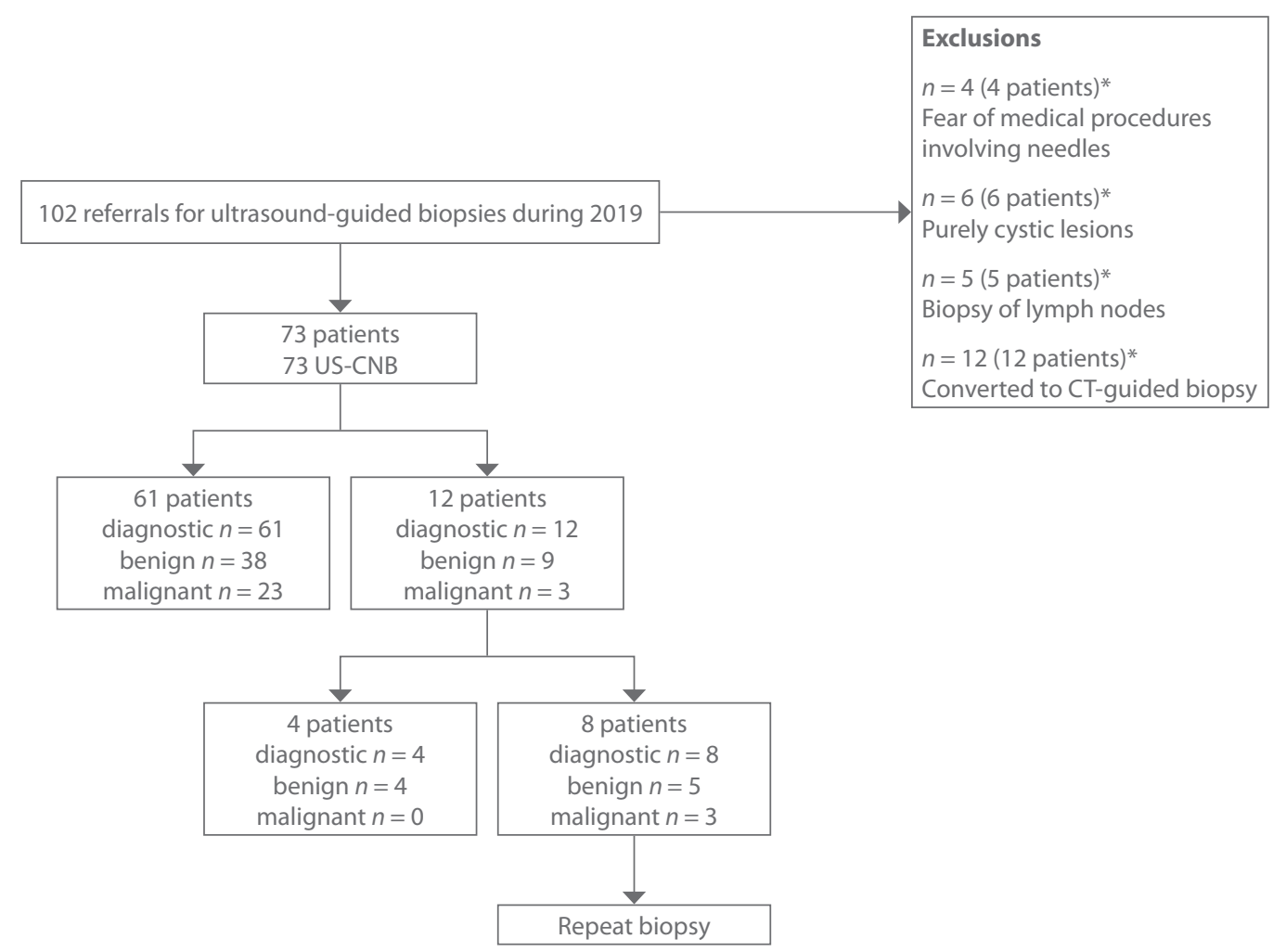

Fig. 1. Flowchart: inclusion and exclusion criteria for the study 


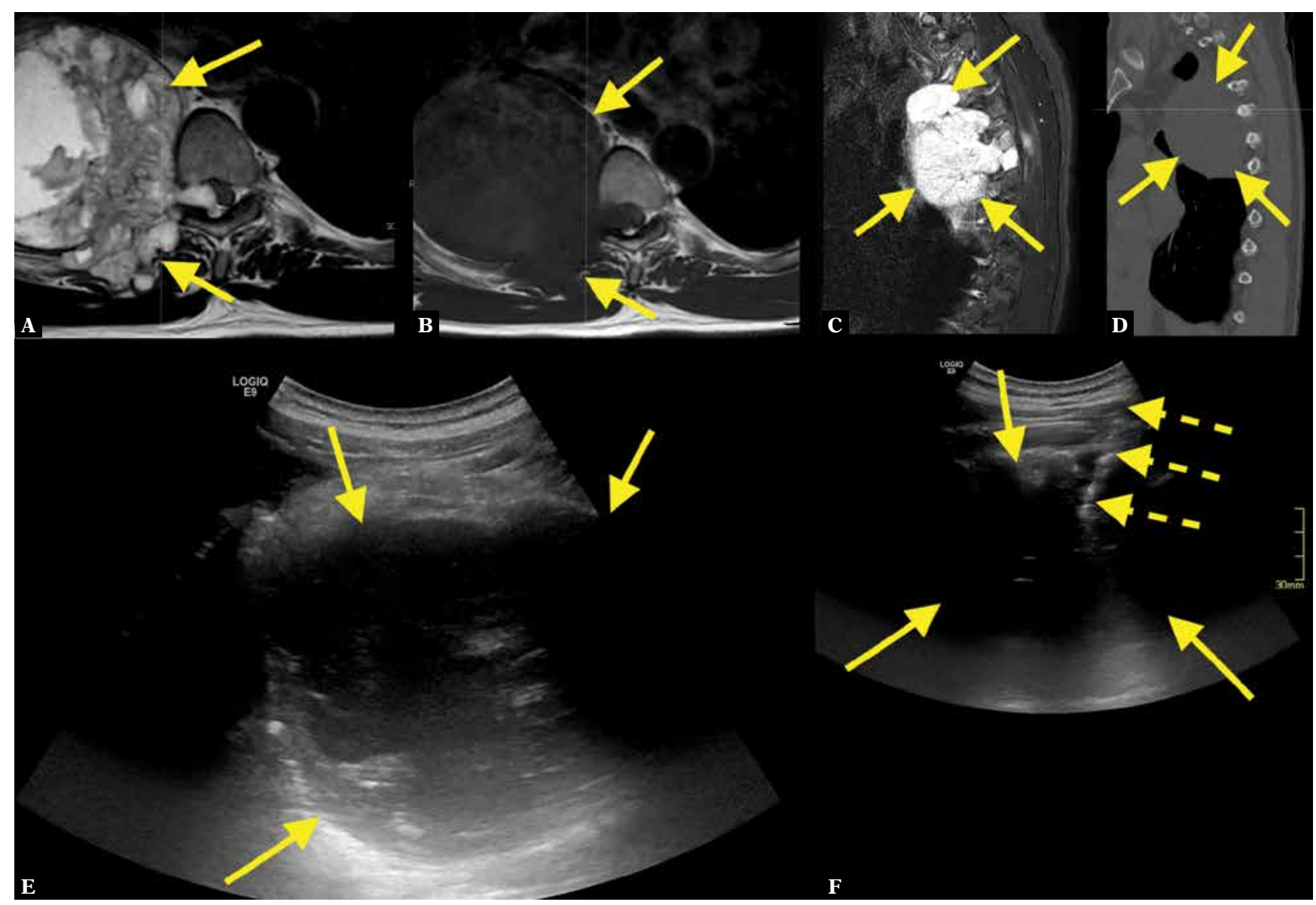

Fig. 2. A 56-year-old patient presented to the emergency room because of pain in the thorax and clonus in the foot. An acute MRI of the whole spine revealed an about $10 \mathrm{~cm}$ long tumor (A-C; straight arrows - tumor). CT showed no calcifications (D). MRI and CT were unspecific, with a wide spectrum of differential diagnoses. US-CNB was performed (E, F; dashed arrows - biopsy needle). A histopathological diagnosis of chondrosarcoma was confirmed after excisional biopsy

from the skin to the lesion was measured on the ultrasound scan. The mean volume of the lesion was calculated by the formula for volume in ultrasound ${ }^{(17)}$. A 16-gauge automatic biopsy needle with a coaxial introducer needle was used. All the procedures were done by radiologists specializing in musculoskeletal radiology. Under local anesthesia (maximum $10 \mathrm{ml}$ Mepivacain $10 \mathrm{mg} / \mathrm{ml}$ ), in sterile conditions, and with ultrasound guidance, at least 2 core biopsy samples were obtained with a minimum length of $1 \mathrm{~cm}$, and fixed in $10 \%$ formaldehyde. All patients were observed for at least 30 minutes after the procedure to check for complications, and received information about possible late complications.

\section{Specimen assessment}

All biopsy specimens were evaluated by pathologists specializing in musculoskeletal pathology at the study hospital. There are no strict definitions related to the evaluation of biopsy effectiveness. In the current study, the following classification was applied: biopsies were classified as diagnostic or not, and accurate or not, based on the pathological findings and the clinical follow-up data. A biopsy was considered diagnostic when a definitive pathological diagnosis could be made from the first biopsy, which was consistent with the clinical findings and follow-up. A biopsy was considered accurate if the tumor could be classified as malignant or benign, and the classification was consistent with subsequent findings from a repeat biopsy, an excisional biopsy, or with the clinical findings, and there was at least 6 months' clinical and radiological follow-up. The overall biopsy success was estimated as the sum of diagnostic biopsies and accurate biopsies, divided by the total number of procedures.

\section{Statistics}

We investigated whether lesions in the groups of accurate and diagnostic biopsies differed regarding calcifications, fat, volume, depth to the skin surface, and their malignant or benign nature.

A $p$-value $<0.05$ was considered statistically significant.

Statistical analysis was performed using SPSS 8.0. 


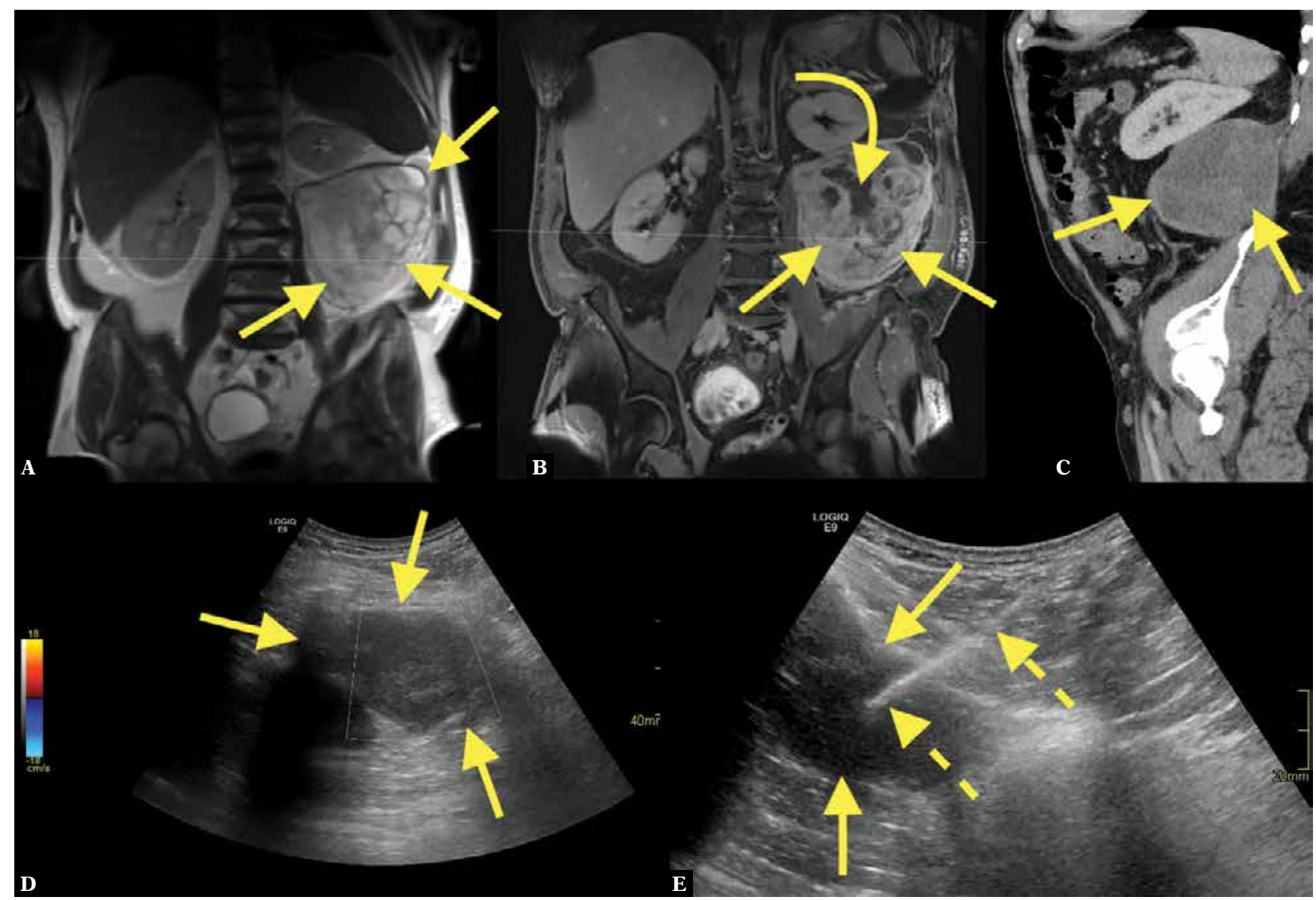

Fig. 3. A 58-year-old patient presented to the emergency room with left-sided abdominal pain. Imaging revealed an about $17 \mathrm{~cm}$ long solid tumor (straight arrows) in the left retroperitoneal space. MRI showed solid and necrotic parts (A, B; curved arrow-solid parts). CT showed the solid tumor in the retroperitoneal space on the left side (C). The patient was referred for US-CNB with suspected liposarcoma. A coaxial US-CNB was performed (D, E). The pathological diagnosis was liposarcoma grade 1

Ethics

Bioethical approval for the current retrospective study was obtained, and the need for informed consent was waived (2020.00158).

\section{Results}

In all 73 patients, US-CNB provided enough tissue to make a histopathological diagnosis in 61 diagnostic biopsies (84\%) (Fig. 2, Fig. 3, Fig. 4, Fig. 5, Fig. 6, Fig. 7, Fig. 8,

Tab. 1. Type of lesion and biopsy success in 73 ultrasound-guided core needle biopsies

\begin{tabular}{|c|c|c|c|c|c|c|}
\hline \multirow{2}{*}{ Biopsy } & \multicolumn{2}{|c|}{$\begin{array}{c}\text { Malignant } \\
\text { lesion } \\
n=26\end{array}$} & \multicolumn{2}{|c|}{$\begin{array}{c}\text { Benign lesion } \\
n=47\end{array}$} & \multicolumn{2}{|c|}{$\begin{array}{c}\text { Overall } \\
n=73\end{array}$} \\
\cline { 2 - 7 } & $\mathrm{n}$ & $\%$ & $\mathrm{n}$ & $\%$ & $\mathrm{n}$ & $\%$ \\
\hline Diagnostic & 23 & $88 \%$ & 38 & $81 \%$ & 61 & $84 \%$ \\
\hline Accurate & 3 & $12 \%$ & 9 & $19 \%$ & 12 & $16 \%$ \\
\hline Inaccurate & 0 & $0 \%$ & 0 & $0 \%$ & 0 & $0 \%$ \\
\hline Successful & 26 & $100 \%$ & 47 & $100 \%$ & 73 & $100 \%$ \\
\hline
\end{tabular}

Tab. 1). The number of diagnostic biopsies was higher in the group of malignant lesions than benign lesions $(88 \%$ vs. $81 \%, p>0.05)$, Tab. 1 . In 12 cases (16\%), the tumor could be characterized as malignant or benign; thus, the biopsy was classified as accurate (Fig. 9, Fig. 10, Fig. 11). The number of accurate biopsies was higher among benign than malignant lesions ( $19 \%$ vs. $12 \%, p>0.05)$, Tab. 1 . The

Tab. 2. Final diagnosis for 12 accurate biopsies

\begin{tabular}{|l|}
\hline Final diagnosis for accurate biopsies in alphabetical order \\
\hline Benign peripheral nerve sheath tumor (Fig. 9) \\
Endometriosis \\
Giant cell tumor \\
Metastatic chondroblastoma \\
Myositis ossificans \\
Nodular fasciitis (Fig. 11) \\
Osteoblastoma (Fig. 10) \\
Unclear case, malign tumor, probably low-grade myxofibrosarcoma, \\
[1 mitosis per 10 high power fields] \\
Benign, mesenchymal tissue, without atypia \\
Vascular malformation (Fig. 8), 2 patients \\
Well-differentiated liposarcoma
\end{tabular}




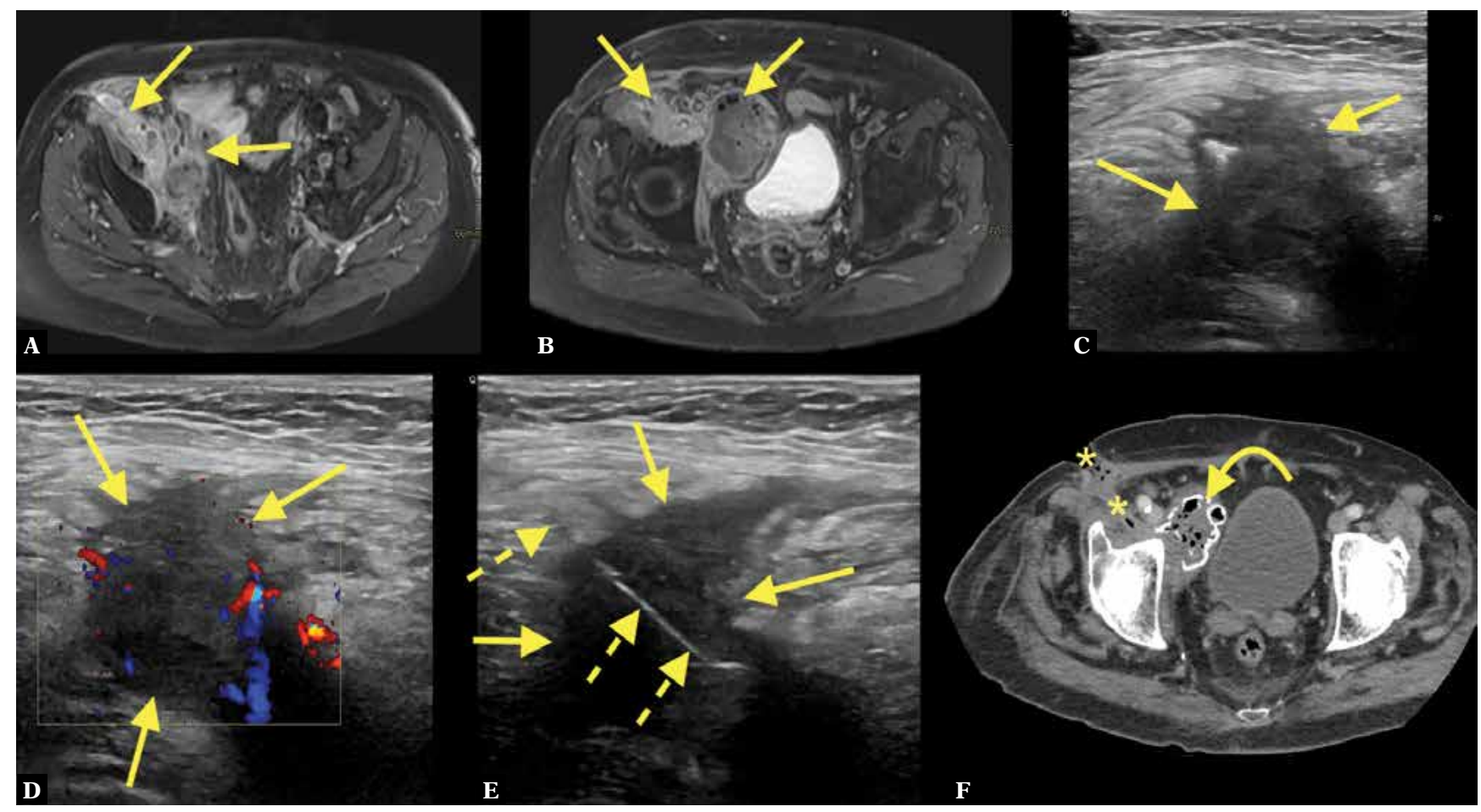

Fig. 4. An 81-year-old patient who three years previously had been operated for an extraskeletal myxoid chondrosarcoma. For the last four months the patient experienced swelling in the operated area, leading to a suspicion of tumor recurrence. MRI (A, B) showed an abnormality in direct relation to the right femoral artery and vein. The patient was referred for US-CNB (C-E). The ultrasound examination revealed a hypoechogenic and vascularized mass. The histopathological diagnosis was inflammation. CT with intravenous contrast performed 2 months after the biopsy showed a fistula lateral to the femoral artery and vein, with an abscess on the medial side, calcifications and gas (F; asterisks - fistula, curved arrow - lesion)

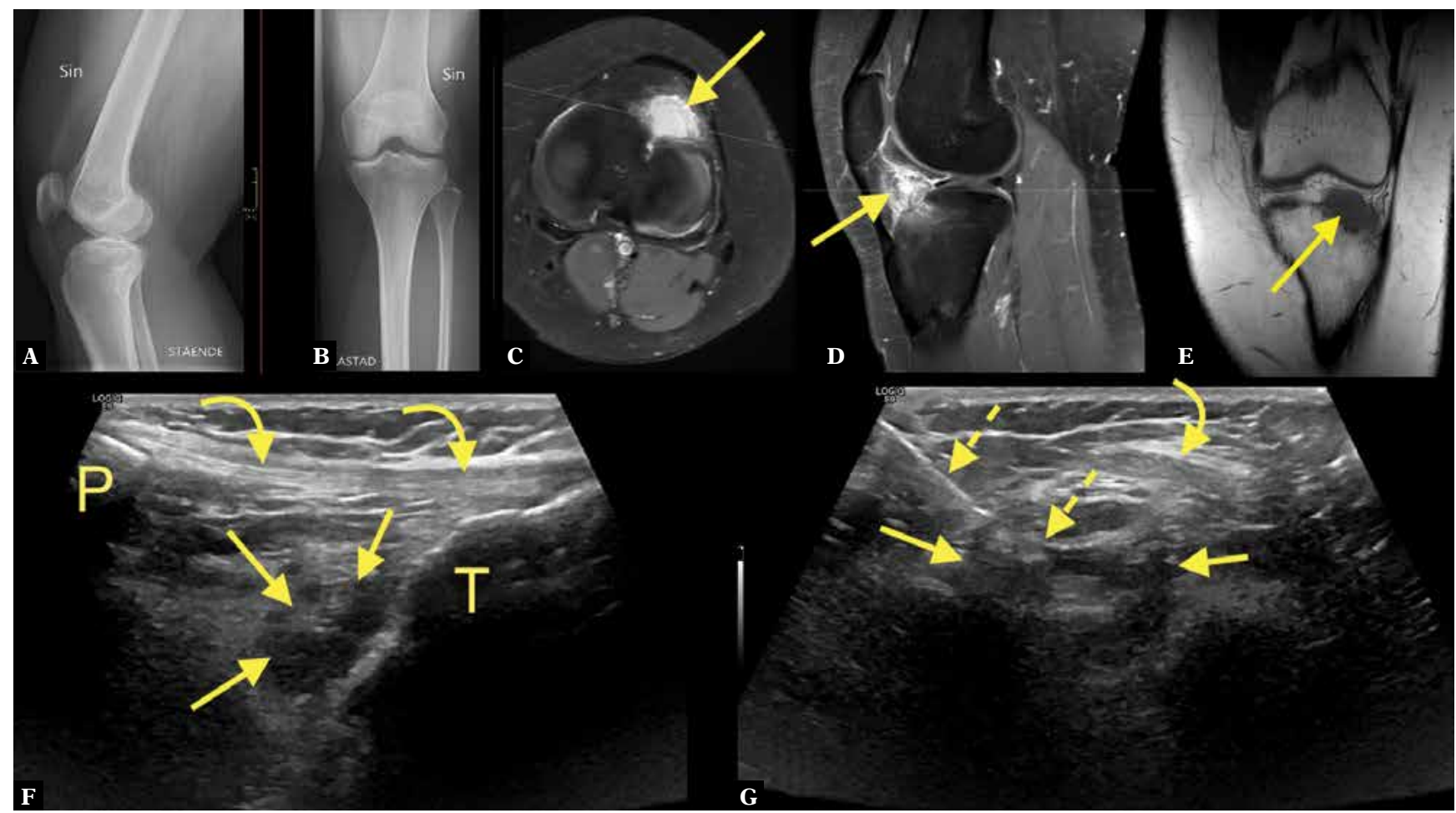

Fig. 5. A 22-year-old patient presenting with knee pain. Radiography (A, B) showed no abnormality. The patient was referred for MRI, which revealed an uncalcified solid contrast-enhancing lesion (C-E; straight arrow-lesion) in the Hoffa fat pad. Localized nodular synovitis was suspected. Because of pain, the patient was referred for US-CNB (F, G; dashed arrow - biopsy needle, curved arrow patellar tendon, $P$ - patella, $T$ - tibia). The pathological diagnosis was lipoma arborescens 


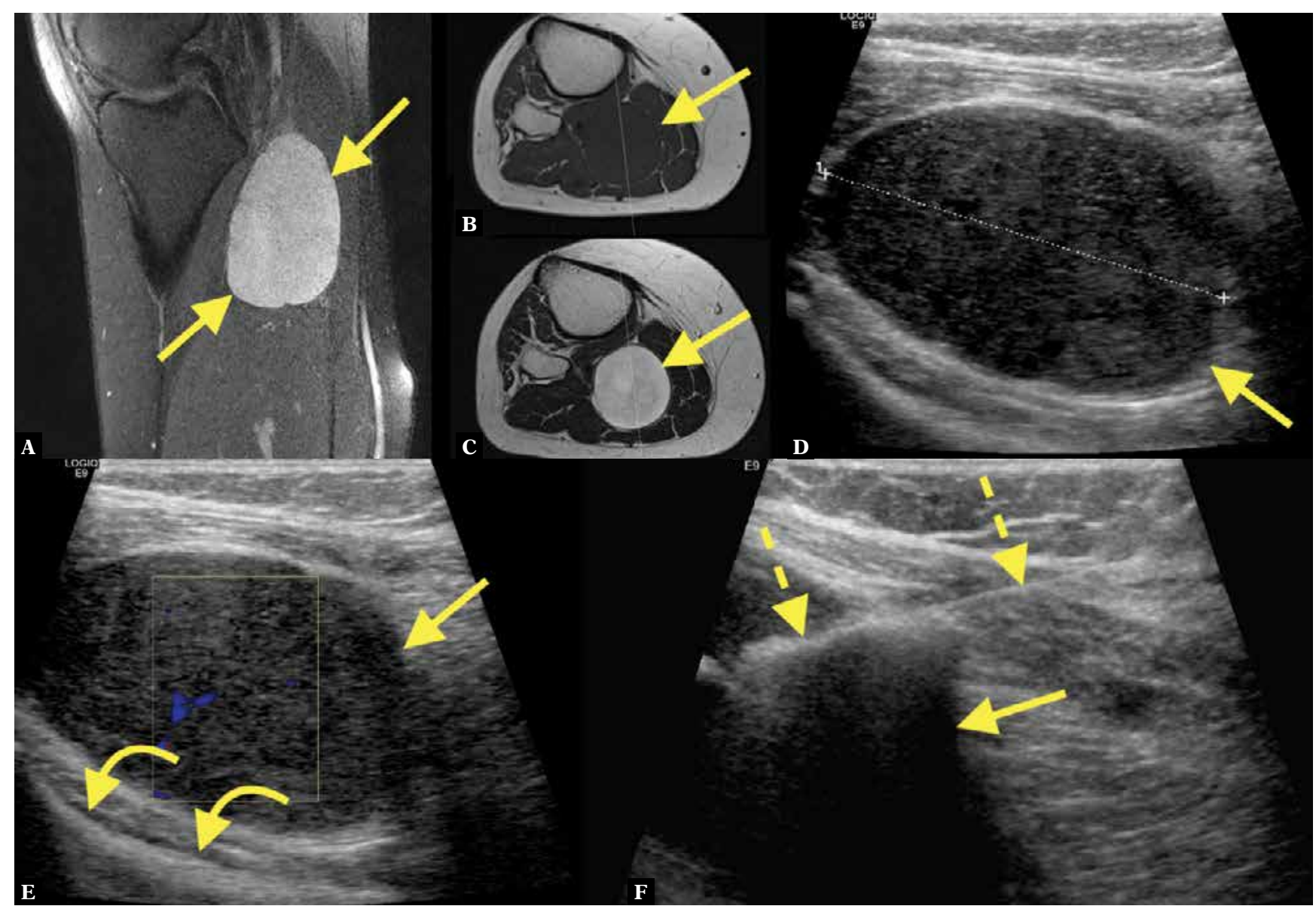

Fig. 6. A 32-year-old patient presented with a painful mass in the popliteal fossa. MRI revealed an intramuscular tumor (A-C; straight arrow - tumor). Ultrasound showed a large hypoechoic mass (D). After suspicion of neurogenic tumor, the patient was referred for US-CNB (E, F; curved arrows - posterior surface of the tibia, dashed arrows - biopsy needle). The histopathological diagnosis was neuroma

discrimination between a malignant and a benign lesion was possible in all the cases included in the current study. Thus, biopsy success in both groups malignant and benign lesions was $100 \%$ (Tab. 1). The patients without a diagnostic biopsy were referred for a new biopsy (Tab. 2).

The study cohort comprised mostly benign lesions (64\%; Tab. 3). The three most common benign lesions were tumor-like lesions (inflammation; Fig. 7), lipoma, and desmoid. Regarding malignant lesions, liposarcoma (Fig. 3) and metastasis were the most common.

Two cases could only be classified as benign or malignant without a definitive pathological diagnosis (Tab. 3). In a single case, there was enough material to determine the benign nature of the lesion; however, no unequivocal final diagnosis could be made, so the biopsy was classified as accurate but not diagnostic. In another case, a low-grade myxofibrosarcoma was considered on histopathology; however, it was inconsistent with other tests (Tab. 1, Tab. 3). The biopsy was repeated without a definitive pathological diagnosis; however, the malignant character was upheld. The working diagnosis became myxofibrosarcoma.
The average minimum distance from the skin to the lesion was $11 \mathrm{~mm}$ (range 2-50 mm), while the mean volume was $70.4 \mathrm{ml}$ (range 0.02-1724 ml). The mean volume of the tumor in accurate biopsies was $154.2 \mathrm{ml}$ vs. $27.8 \mathrm{ml}$ in diagnostic biopsies ( $p<0,001$, Tab. 4). No statistically significant differences were found between the number of diagnostic biopsies depending on age, gender, presence of fat, calcifications, depth of the lesion, and malign or benign character of the lesion (Tab. 4). The number of diagnostic and accurate biopsies did not vary significantly between different anatomical locations of the lesion (Tab. 5).

\section{Discussion}

Appropriate treatment of soft tissue tumors in the musculoskeletal system requires an unquestionable diagnosis. In many cases, the radiological image may not be typical or might not match the clinical symptoms. In such situations, histopathological confirmation of the diagnosis is necessary ${ }^{(7,13)}$. US-CNB is suitable for making a diagnosis in such borderline or unclear cases where the radiological signs or clinical symptoms are ambiguous ${ }^{(2,7)}$. 


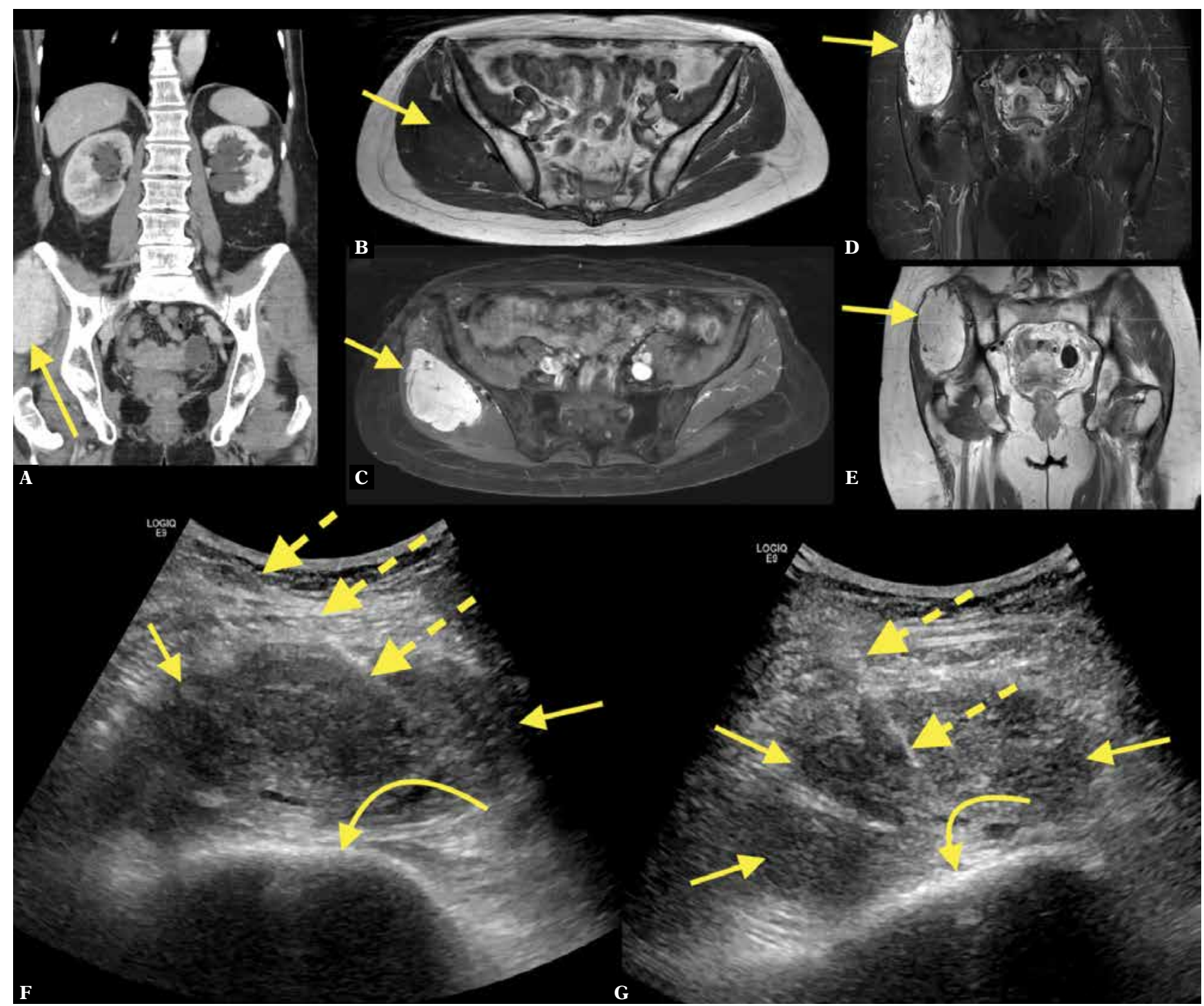

Fig. 7. A 70-year-old patient with a history of malignant melanoma operated 3 years before presented with pain in the abdomen and right buttock. CT with intravenous contrast in the portal venous phase showed an intramuscular contrast-enhancing mass on the right (A; straight arrow-mass). MRI of the pelvis with intravenous contrast showed a solid contrast-enhancing tumor in the gluteal muscles (B-E). MRI and CT were unspecific, with a wide spectrum of differential diagnoses. The patient was referred for US-CNB (F, G; dashed arrows - biopsy needle, curved arrow - iliac bone). The histopathological diagnosis was extrapleural solitary fibrous tumor

A final diagnosis, without differential diagnoses (called a diagnostic biopsy), allows the clinician to decide on the patient's treatment, to dismiss or follow up ${ }^{(18)}$. This does not mean that other types of biopsy results are without value ${ }^{(19-21)}$. In some cases, it is possible to determine only the benign or malignant nature of the lesion (called accurate biopsy)(18), which, in combination with clinical data, provides relevant information that may influence the further management of the patient ${ }^{(20,21)}$. The ability to differentiate malignant tumors from benign masses based on the results of percutaneous ultrasound-guided biopsy is mostly reported as ranging from $68 \%$ to $96 \%$ (accurate biopsy) ${ }^{(8-11,18-23)}$. The overall biopsy success in the current study did not differ significantly from the results reported by other authors, at the level of $83-97 \%(3,9,10,12,20)$. However, a detailed comparison with previously published reports is difficult because of differences in group selection and size, and localization and character of lesions ${ }^{(9,10,24,25)}$. The current study included only patients with solid lesions in the soft tissues, with biopsies performed with a 16-gauge needle. Previously published reports have included lesions partially located in the bone or bony lesions - or cases where different sizes of needles were used.

Most of previously published reports rely on retrospective data, so similarly to the current study, information about the exact quantity of the tissue samples taken during biopsy may be lacking. There are also differences in the needles used during biopsy. Some studies have used 14-gauge to 23 -gauge biopsy needles ${ }^{(8,10,19)}$, while in the current study all biopsies were performed with a 16-gauge needle. 


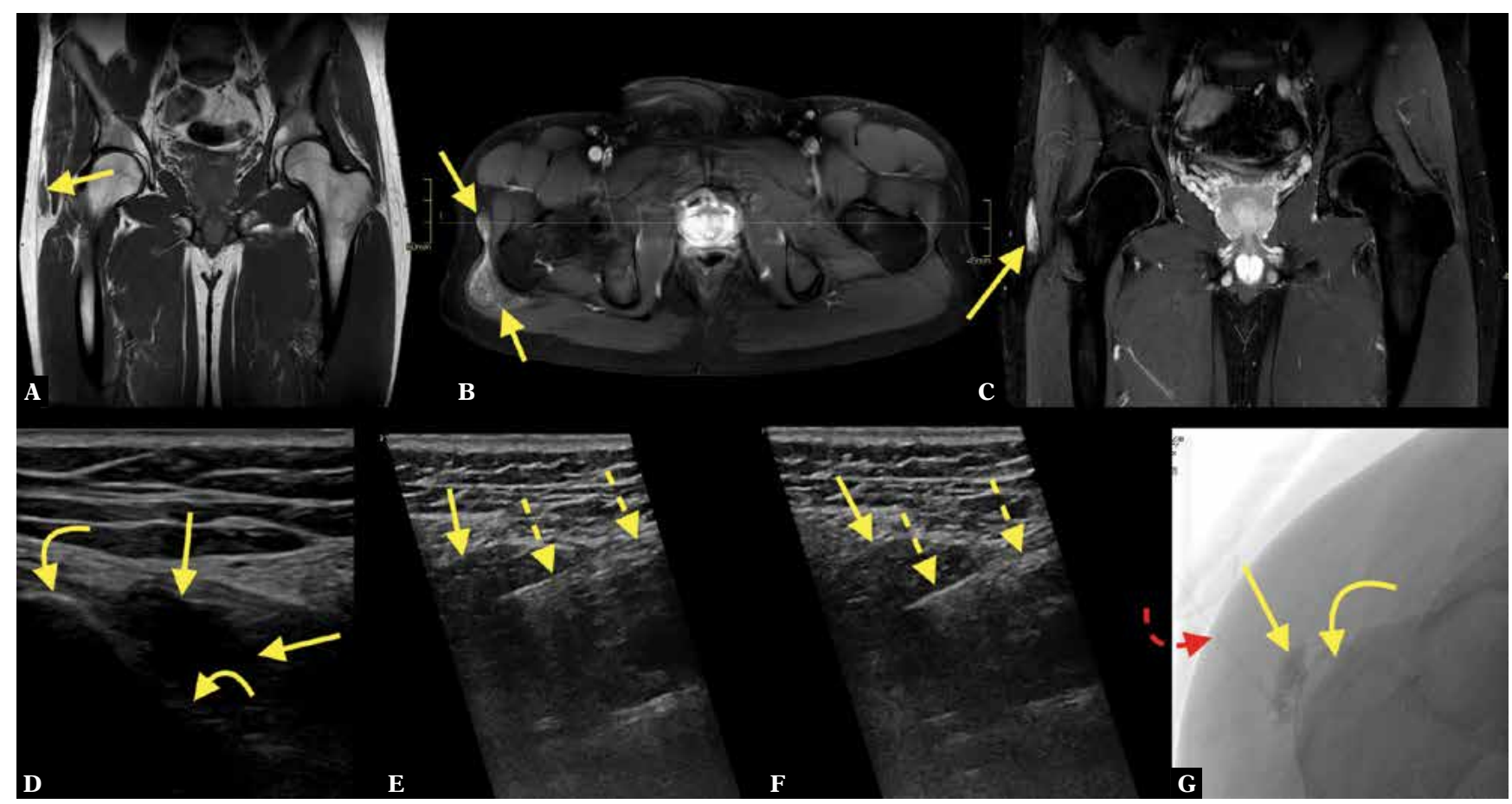

Fig. 8. A 28-year-old patient presented with a chronically swollen trochanteric region and more discomfort during the last weeks. There was a clinical suspicion of greater trochanteric bursitis. The patient was referred for MRI, where a solid lesion, not bursitis, in close relation to the greater trochanter, was detected (A; straight arrow - lesion). After intravenous contrast administration (B, C) there was vivid contrast enhancement. No muscle atrophy was visible. The MRI was unspecific, with a wide spectrum of lesions in the differential diagnosis. The patient was referred for US-CNB (D-F; curved arrows - greater trochanter, dashed arrow-biopsy needle). A histopathological diagnosis of a benign lesion, with a suspicion of vascular malformation, was made, and the patient was referred for intravascular treatment (G; red dashed curved arrow - catheter) which produced a good effect

US-CNB allows the safe passage of the biopsy needle even into small and difficult-to-reach lesions, so that damage to important structures including the nerves or vessels can be avoided ${ }^{(7,20)}$. Ultrasound guidance has a well-established position in the multidisciplinary diagnosis of musculoskeletal lesions because of its high accuracy and low complication rate. However, the success of a biopsy seems to be variable and depending on several factors such as operator expertise, tumor structure, amount of retrieved material, needle diameter, and patient selection ${ }^{(2,9,18)}$.

In our study, we demonstrated a difference in tumor volume between the accurate and diagnostic biopsy groups. We found a significantly higher volume of tumor in the group of accurate biopsies compared to diagnostic biopsies. This may seem paradoxical, but larger tumors tend to be more heterogeneous, with more necrosis, making it more challenging to collect an appropriate tissue sample. The procedure of tissue sample collection from larger and more heterogeneous tumors can be challenging, as it requires performing a biopsy in different directions to obtain samples from various parts of the tumor. It is easier to assess the structure of more solid and homogenous tumors on ultrasound and thus simpler to perform a biopsy, with better yield ${ }^{(8-10)}$. However, there are some studies of non-musculoskeletal tumors where the size of the lesion was not correlated with biopsy success ${ }^{(26)}$. Patient selection and the smaller number of patients in the group of malignant tumors may also have affected our observations.
There are some disadvantages of US-CNB compared to open biopsy ${ }^{(3,12,24)}$. The main disadvantage is the smaller amount of retrieved tissue, which may require additional tests to obtain the final diagnosis ${ }^{(20)}$. We have not shown any significant differences in tumor depth between the groups of benign and malignant lesions. With more deeply located tumors, it is harder to obtain a good quality of tissue sample using ultrasound; therefore CT-guided biopsy should be taken into consideration in such cases ${ }^{(9,19)}$. In previous studies, a high value of biopsy has been shown even for lesions smaller than $2 \mathrm{~cm}^{(24)}$. A perusal of the literature has not revealed any previous reports correlating biopsy success or accuracy with lesion volume. Most typically, only one dimension is used, which does not fully characterize the tumor ${ }^{(24)}$.

The presence of calcifications or fat in the tumor may have an influence on tumor visualization on ultrasound, which may correlate with the ability to collect appropriate tissue material. In the current study, the incidence of diagnostic biopsy was higher, though without statistical significance, in the group of malignant lesions, which is in contrast with previously published results ${ }^{(10,12,20)}$.

Diagnostic imaging is crucial when considering lesions for imaging-guided biopsy. Some benign lesions may mimic malignant lesions at the microscopic examination. An example of such a lesion is myositis ossificans, which may resemble osteosarcoma ${ }^{(27)}$ in the histopathological 


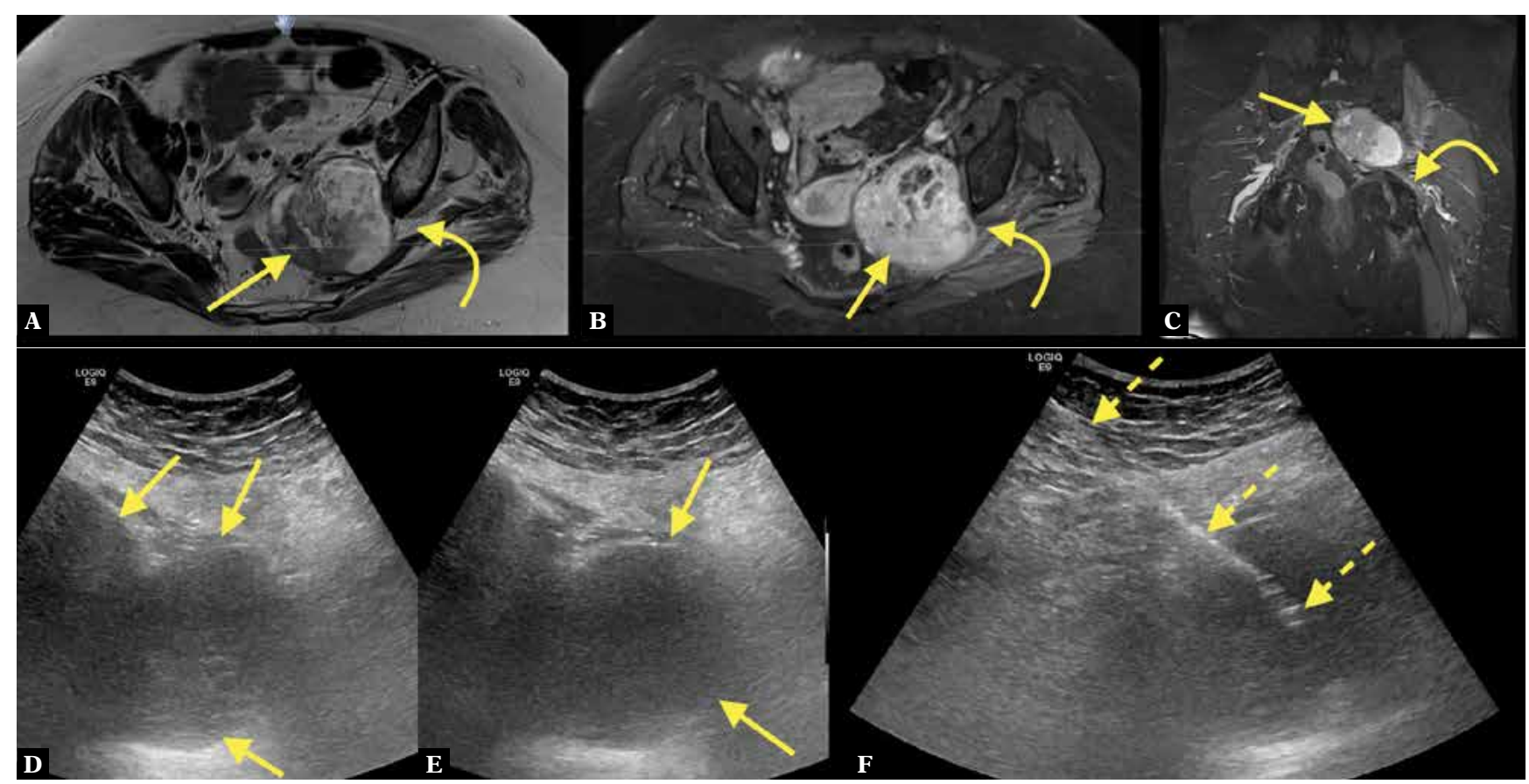

Fig. 9. An 83-year-old patient with pain in the left buttock and leg. MRI of the pelvis with intravenous contrast revealed a tumor in the greater sciatic foramen (A-C; straight arrow-tumor, curved arrow - sciatic nerve). The MRI was unspecific; however, a diagnosis of malignant peripheral nerve sheath tumor was made because of the tumor's direct relation to the sciatic nerve. The patient was referred for US-CNB (D-F; dashed arrows - biopsy needle). No histopathological diagnosis could be made. The patient was referred once again for US-CNB, where the histopathological diagnosis of benign peripheral nerve sheath tumor was made and confirmed after excisional biopsy

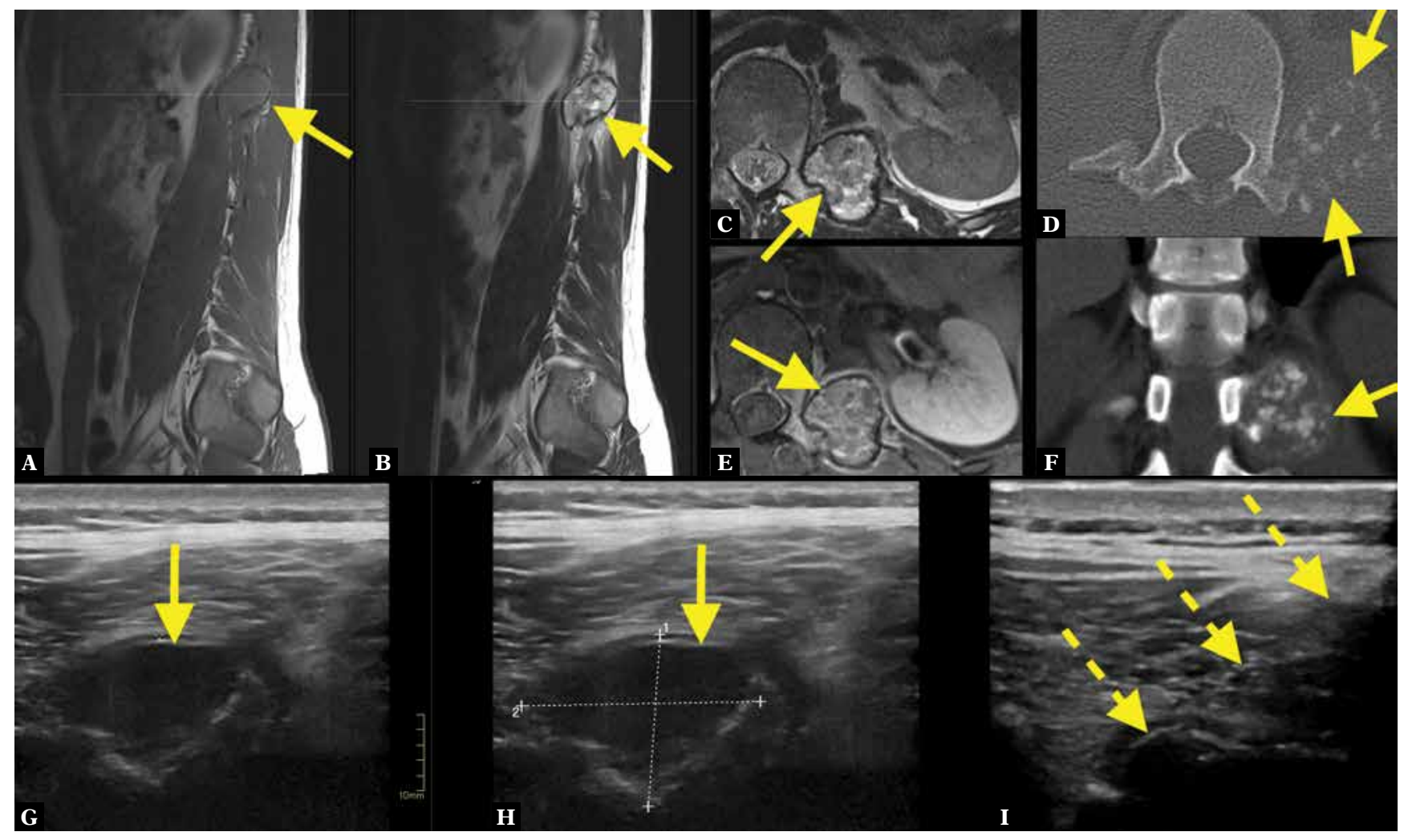

Fig. 10. A 20-year-old football player with pain in the thoracic spine. The patient was referred for spinal MRI, where a solid tumor with calcifications was revealed on the left side of Th12 (A-C, E; straight arrow-tumor). CT without intravenous contrast (D, F) showed calcifications both in the center and periphery of the lesion, which in combination with the patient's pain was worrisome. The differential diagnosis included myositis ossificans, osteosarcoma, and osteoblastoma. US-CNB was performed (G-I; dashed arrows - biopsy needle). The final histopathological diagnosis of osteoblastoma was made on a repeated US-CNB 


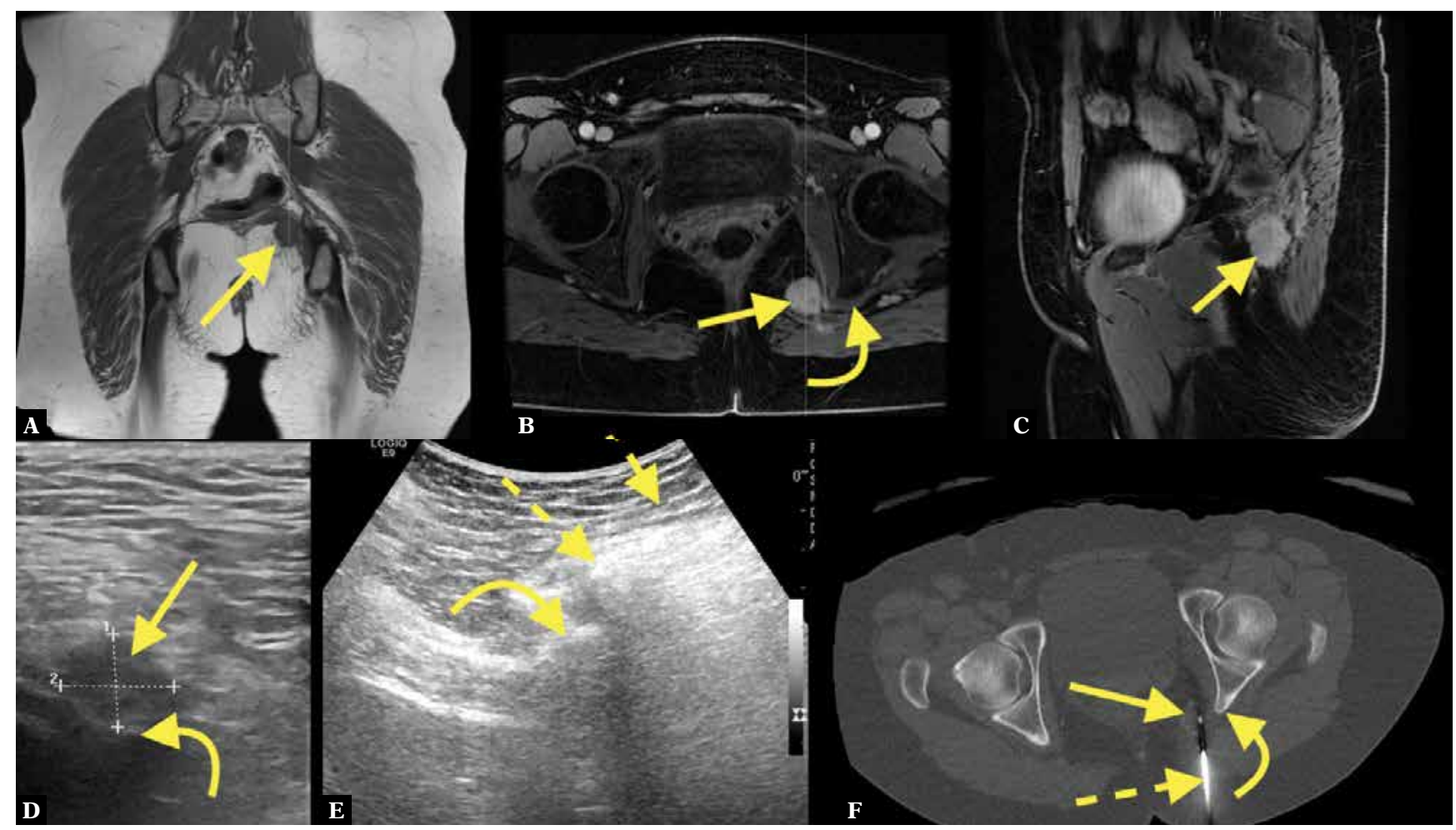

Fig. 11. A 41-year-old female presenting with pain at the left ischial tuberosity. MRI of the pelvis with intravenous contrast revealed a tumor, about $15 \mathrm{~mm}$ long, in relation to the left sciatic spine (A-C; curved arrow-tumor). MRI and CT were unspecific, with a wide spectrum of lesions in the differential diagnosis. US-CNB (D, E) was performed without a final histopathological diagnosis of a benign lesion. After a CT-guided biopsy (F) the final histopathological diagnosis was nodular fasciitis

examination, but the radiological image is more characteristic. Therefore, the radiologist's role is to prevent an unnecessary biopsy when a typical radiological image is obtained.

There are some limitations of the current study, such as its retrospective character. Furthermore, only some of the patients were operated on, so the results of excisional biopsies are available in only a few cases. Finally, information about the quantity and length of the tissue sample was often missing.

\section{Conclusions}

In conclusion, the diagnostic value of ultrasound-guided percutaneous core needle biopsy of musculoskeletal soft tissue lesions performed with 16-gauge needle is good, with a high rate of diagnostic biopsies, both for benign and malignant lesions. Ultrasound-guided core biopsy of solid musculoskeletal lesions is an important tool to obtain tissue samples after an appropriate selection of patients based on previous $\mathrm{CT}$ or MRI evaluation. Factors including the presence of fat, calcification, benign or malignant character of the tumor, depth or anatomical localization do not differ significantly between groups of accurate and diagnostic biopsies. Tumor volume varied between the study groups, and was higher in the group of accurate biopsies. A multidisciplinary approach to the diagnosis of soft tissue tumors facilitates an appropriate selection of patients for this procedure.
Tab. 3. Final histopathology results in 73 ultrasound-guided core needle biopsies, ordered by quantity

\begin{tabular}{|c|c|c|c|}
\hline \multicolumn{2}{|l|}{ Benign } & \multicolumn{2}{|l|}{ Malignant } \\
\hline Inflammation & 8 & Well-differentiated liposarcoma & 7 \\
\hline Lipoma & 6 & Chondrosarcoma & 2 \\
\hline Desmoid & 5 & Metastatic gastric cancer & 2 \\
\hline Vascular malformation & 4 & $\begin{array}{l}\text { Metastatic malignant me- } \\
\text { lanoma }\end{array}$ & 2 \\
\hline Intramuscular lipoma & 3 & $\begin{array}{l}\text { Pleomorphic spindle cell } \\
\text { sarcoma (high grade) }\end{array}$ & 2 \\
\hline Myxoma & 3 & Dedifferentiated liposarcoma & 1 \\
\hline Neurofibroma & 2 & Leiomyosarcoma & 1 \\
\hline $\begin{array}{l}\text { Pigmented villonodular } \\
\text { synovitis }\end{array}$ & 2 & Lymphoma & 1 \\
\hline Atheroma & 1 & Metastatic breast cancer & 1 \\
\hline Endometriosis & 1 & Metastatic chondroblastoma & 1 \\
\hline Fibroma & 1 & Metastatic lung cancer & 1 \\
\hline Giant cell tumor $(\mathrm{CT})$ & 1 & Metastatic prostate cancer & 1 \\
\hline Hematoma & 1 & $\begin{array}{l}\text { Metastatic squamous cell } \\
\text { carcinoma }\end{array}$ & 1 \\
\hline Hibernoma & 1 & Myeloma/plasmocytoma & 1 \\
\hline Lipoma arborescens & 1 & Recurrent Ewing's sarcoma & 1 \\
\hline Myositis ossificans (CT) & 1 & \multicolumn{2}{|r|}{$n=25$} \\
\hline Nodular fasciitis (CT) & 1 & \multicolumn{2}{|l|}{ Unclear } \\
\hline Osteomyelitis & 1 & $\begin{array}{l}\text { Benign, mesenchymal tissue, } \\
\text { without atypia }\end{array}$ & 1 \\
\hline Schwannoma & 1 & $\begin{array}{l}\text { Malign, unclear case, pro- } \\
\text { bably low-grade myxofibro- } \\
\text { sarcoma }\end{array}$ & 1 \\
\hline Solitary fibrous tumor & 1 & $\begin{array}{l}\text { [1 mitosis per } 10 \text { high power } \\
\text { fields] }\end{array}$ & \\
\hline Vascular hamartoma & 1 & \multicolumn{2}{|r|}{$n=2$} \\
\hline & $=4$ & & $n=73$ \\
\hline
\end{tabular}


Tab. 4. Differences between age, gender, tumor character, presence of fat and calcifications, volume and depth in accurate and diagnostic biopsies

\begin{tabular}{|c|c|c|c|}
\hline & Accurate & Diagnostic & $p$ \\
\hline Average age [years] & 47 & 60 & \multirow{4}{*}{0.13} \\
\hline SD & 26.1 & 18.2 & \\
\hline $\min$ & 8 & 15 & \\
\hline $\max$ & 75 & 89 & \\
\hline Male $[n]$ & 6 & 31 & \multirow{2}{*}{0.96} \\
\hline$\%$ & $8.2 \%$ & $42.5 \%$ & \\
\hline Benign [ $n]$ & 9 & 38 & \multirow{2}{*}{0.35} \\
\hline$\%$ & $12.3 \%$ & $52.1 \%$ & \\
\hline Malign [n] & 3 & 23 & \multirow{2}{*}{0.28} \\
\hline$\%$ & $4.1 \%$ & $31.5 \%$ & \\
\hline Fat $[n]$ & 2 & 13 & \multirow{2}{*}{0.44} \\
\hline$\%$ & $2.7 \%$ & $17.8 \%$ & \\
\hline Calcification $[n]$ & 3 & 3 & \multirow{2}{*}{0.27} \\
\hline$\%$ & $4.1 \%$ & $4.1 \%$ & \\
\hline Volume $\left[\mathrm{cm}^{3}\right]$ & 154.2 & 27.8 & \multirow{4}{*}{$<0.001$} \\
\hline SD & 494.7 & 64.0 & \\
\hline $\min$ & 0.3 & 0.02 & \\
\hline $\max$ & 1724.8 & 415.8 & \\
\hline Depth [mm] & 12.8 & 9.7 & \multirow{4}{*}{0.18} \\
\hline SD & 7.6 & 7.8 & \\
\hline $\min$ & 4 & 1 & \\
\hline $\max$ & 27.4 & 31.5 & \\
\hline
\end{tabular}

\section{References}

1. Kilpatrick SE, Cappellari JO, Bos GD, Gold SH, Ward WG: Is fineneedle aspiration biopsy a practical alternative to open biopsy for the primary diagnosis of sarcoma? Experience with 140 patients. Am J Clin Pathol 2001; 115: 59-68.

2. Mankin HJ, Mankin CJ, Simon MA: The hazards of the biopsy, revisited. Members of the Musculoskeletal Tumor Society. J Bone Joint Surg Am 1996; 78: 656-663.

3. Kasraeian S, Allison DC, Ahlmann ER, Fedenko AN, Menendez LR: A comparison of fine-needle aspiration, core biopsy, and surgical biopsy in the diagnosis of extremity soft tissue masses. Clin Orthop Relat Res 2010; 468: 2992-3002.

4. El Saghir NS, Keating NL, Carlson RW, Khoury KE, Fallowfield L: Tumor boards: optimizing the structure and improving efficiency of multidisciplinary management of patients with cancer worldwide. Am Soc Clin Oncol Educ Book 2014: e461-e466.

5. Blay J-Y, Soibinet P, Penel N, Bompas E, Duffaud F, Stoeckle E et al.: Improved survival using specialized multidisciplinary board in sarcoma patients. Ann Oncol 2017; 28: 2852-2859.

6. Siegel GW, Biermann JS, Chugh R, Jacobson JA, Lucas D, Feng M et al.: The multidisciplinary management of bone and soft tissue sarcoma: an essential organizational framework. J Multidiscip Healthc 2015; 8: 109-115.

7. Gogna A, Peh WC, Munk PL: Image-guided musculoskeletal biopsy. Radiol Clin North Am 2008; 46: 455-473, v.

8. Lopez JI, Del Cura JL, Zabala R, Bilbao FJ: Usefulness and limitations of ultrasound-guided core biopsy in the diagnosis of musculoskeletal tumours. APMIS 2005; 113: 353-360.

9. Sung KS, Seo SW, Shon MS: The diagnostic value of needle biopsy for musculoskeletal lesions. Int Orthop 2009; 33: 1701-1706.

10. Torriani M, Etchebehere M, Amstalden E: Sonographically guided core needle biopsy of bone and soft tissue tumors. J Ultrasound Med 2002; 21: $275-281$.
Tab. 5. Distribution of lesions in 73 ultrasound-guided core needle biopsies

\begin{tabular}{|l|c|}
\hline Upper extremity & 26 \\
\hline Shoulder & 11 \\
Upper arm & 7 \\
Elbow & 3 \\
Forearm & 5 \\
\hline Lower extremity & 35 \\
\hline Pelvis and hip & 13 \\
Thigh & 12 \\
Knee & 5 \\
Lower leg & 4 \\
Ankle & 1 \\
\hline Trunk & 12 \\
\hline Chest wall & 5 \\
Abdominal wall and retroperitoneal space & 4 \\
Paravertebral soft tissue & 3 \\
\hline Total & 73 \\
\hline
\end{tabular}

\section{Conflict of interest}

Authors do not report any financial or personal connections with other persons or organizations which might negatively affect the contents of this publication and/or claim authorship rights to this publication.

11. Le HB, Lee ST, Munk PL: Image-guided musculoskeletal biopsies. Semin Intervent Radiol 2010; 27: 191-198.

12. Mitsuyoshi G, Naito N, Kawai A, Kunisada T, Yoshida A, Yanai H et al.: Accurate diagnosis of musculoskeletal lesions by core needle biopsy. J Surg Oncol 2006; 94: 21-27.

13. Kilpatrick SE, Geisinger KR: Soft tissue sarcomas: the usefulness and limitations of fine-needle aspiration biopsy. Am J Clin Pathol 1998; 110: 50-68.

14. Welker JA, Henshaw RM, Jelinek J, Shmookler BM, Malawer MM: The percutaneous needle biopsy is safe and recommended in the diagnosis of musculoskeletal masses. Cancer 2000; 89: 2677-2686.

15. Oliveira MP, Lima PM, da Silva HJ, de Mello RJ: Neoplasm seeding in biopsy tract of the musculoskeletal system. A systematic review. Acta Ortop Bras 2014; 22: 106-110.

16. Shyamala K, Girish HC, Murgod S: Risk of tumor cell seeding through biopsy and aspiration cytology. J Int Soc Prev Community Dent 2014; 4: 5-11.

17. Hvarness H, Skjoldbye B, Jakobsen H: Urinary bladder volume measurements: comparison of three ultrasound calculation methods. Scand J Urol Nephrol 2002; 36: 177-181.

18. Omura MC, Motamedi K, UyBico S, Nelson SD, Seeger LL: Revisiting CT-guided percutaneous core needle biopsy of musculoskeletal lesions: contributors to biopsy success. AJR Am J Roentgenol 2011; 197: 457-461.

19. Yeow KM, Tan CF, Chen JS, Hsueh C: Diagnostic sensitivity of ultrasound-guided needle biopsy in soft tissue masses about superficial bone lesions. J Ultrasound Med 2000; 19: 849-855.

20. Konermann W, Wuisman P, Ellermann A, Gruber G: Ultrasonographically guided needle biopsy of benign and malignant soft tissue and bone tumors. J Ultrasound Med 2000; 19: 465-471.

21. Abd Elmageed MK, Abd Alwahed MS, Anas Zytoon A: Ultrasound and CT guided biopsy of suspicious musculoskeletal lesions: diagnostic performance and implications for the management. IJRRT 2020; 7: 16-22. 
22. Diniz Ferreira FBM, Bertin SK, Nico M, Gonzalez MT, Souza MR, Garcia DAL et al.: Musculoskeletal imaging-guided biopsies: assessment of techniques and applicability. Current Radiology Reports 2017; 5.

23. Yoon MA, Chung HW, Chee CG, Lee MH, Lee SH, Shin MJ: Risk factors for diagnostic failure of ultrasound-guided core needle biopsy of softtissue tumors based on World Health Organization classification category and biologic potential. AJR Am J Roentgenol 2020; 214: 413-421.

24. Kim SY, Chung HW: Small musculoskeletal soft-tissue lesions: US-guided core needle biopsy-comparative study of diagnostic yields according to lesion size. Radiology 2016; 278: 156-163.
25. Khoo M, Pressney I, Hargunani R, Saifuddin A: Small, superficial, indeterminate soft-tissue lesions as suspected sarcomas: is primary excision biopsy suitable? Skeletal Radiol 2017; 46: 919-924.

26. Desportes E, Wagner M, Kamal M, Salomon AV, Deniziaut G, Pierron G et al.: Prognostic factors of successful on-purpose tumor biopsies in metastatic cancer patients included in the SHIVA prospective clinical trial. Oncotarget 2017; 8: 1760-1773.

27. Schmitz KJ, Losch M, Agaimy A: Myositis ossificans mimicking metaplastic breast cancer on core needle biopsy. Hum Pathol 2019; 93: 97-102. 